\title{
Desempenho de frangos de corte alimentados com dietas contendo diformiato de potássio
}

\author{
Broilers performance fed with diets contend potassium diformiate
}

\author{
Samuel Augusto dos Santos ${ }^{\mathrm{I}}$ Régis Fernando Pastorelo Meurer ${ }^{\mathrm{I}}$ Marcelo França $^{\mathrm{I}}$ \\ Alex Maiorka ${ }^{I *}$ Édson Gonçalves de OliveiraII Ana Vitória Fisher da Silva ${ }^{\text {II }}$ \\ Sebastião Aparecido Borges ${ }^{\mathrm{IV}}$
}

RESUMO

A proibição do uso de antibióticos como aditivos promotores de crescimento causou grandes impactos à avicultura de corte, trazendo consigo a necessidade do desenvolvimento de novos aditivos. Nesse contexto, o diformiato de potássio (DK), um sal de ácido fórmico, surge como uma dessas alternativas. O presente estudo foi realizado com o objetivo de avaliar a ação do DK sobre o desempenho de frangos de corte. Foram utilizados 1.800 frangos de corte machos da linhagem Ross 308, distribuídos em dois experimentos de 900 aves cada, formados por cinco tratamentos de seis repetições, em um delineamento inteiramente ao acaso. Os tratamentos foram constituídos pela inclusão de níveis crescentes de $D K$ às dietas $\left(0,3,6,9\right.$ e $\left.12 \mathrm{~kg} \mathrm{DK} \mathrm{t}^{-1}\right)$. As aves foram pesadas ao alojamento, aos 21 e aos 42 dias $e$, posteriormente, os dados obtidos foram submetidos a uma análise de regressão linear. No primeiro experimento, não houve melhoria no desempenho zootécnico das aves, havendo ainda uma redução no consumo alimentar. Da mesma forma, no segundo experimento a adição de $D K$ reduziu linearmente o consumo de ração e ganho de peso, não demonstrando qualquer efeito sobre a conversão alimentar das aves. Conclui-se que doses elevadas de DK causam desequilíbrio ácido-básico das dietas, com efeitos negativos sobre o desempenho das aves.

Palavras-chave: ácido-base, balanço eletrolítico, desempenho zootécnico.

\section{ABSTRACT}

The use of prohibited antibiotics as growth promoters additives caused great impacts on poultry, bringing the need for the development of new additives. The diformiate potassium (DK), a salt of formic acid, is emerging as one of those alternatives. This experiment was conducted to evaluate the action of DK on the performance of broilers. One thousand and eight hundred broilers male of Ross 308 breed, distributed into two trials of 900 birds each, and then randomly separated by five treatments with six replications, in a completely randomized experimental design, The treatments consisted by inclusion of increasing levels of $D K$ to the diets $(0,3,6,9$ and $\left.12 \mathrm{~kg} \mathrm{DK}^{-1}\right)$. In the first trial carried out, the birds were fed with diets containing $D K$ during the period 1 to 42 days. In the second trial, DK received only in the period from 21 to 42 days old. In both, the birds were weighed to housing, at 21 and 42 days and then the data were subjected to a linear regression analysis. In the first experiment, there was no improvement in the performance of birds, and there was a reduction in feed intake. Similarly, in the second trial, the addition of DK reduced linearly the feed intake and weight gain, however, any effect on feed conversion in poultry. It was concluded that high dosis of DK cause acid-base imbalance of diets, with negative effects on the birds performance.

Key words: acid-base, electrolyte balance, performance.

\section{INTRODUÇÃO}

A intensificação das pesquisas a cerca da adequação das dietas para frangos de corte às reais necessidades das aves tem contribuído para o desenvolvimento de novos aditivos, capazes de aumentar a eficiência de utilização dos nutrientes das

\footnotetext{
IPós-graduação em Ciências Veterinárias, Universidade Federal do Paraná (UFPR), Curitiba, PR, Brasil.

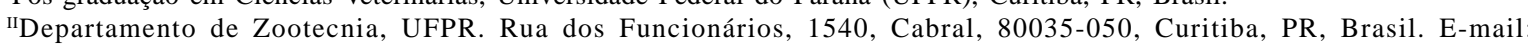
amaiorka@ufpr.br.*Autor para correspondência.

IIIDepartamento de Fisiologia, UFPR, Curitiba, PR, Brasil.

${ }^{\text {IV }}$ Departamento de Zootecnia, Universidade Tuiuti do Paraná (UTP), Curitiba, PR, Brasil.
} 
dietas pelas aves. A recente proibição do uso de antibióticos como aditivos promotores de crescimento imposta pelos principais importadores da carne de frango brasileira é outro fator que impulsiona o desenvolvimento de aditivos, fomentando a competitividade da avicultura brasileira no cenário internacional.

Dentre os vários produtos que vêm sendo estudados, figuram os ácidos orgânicos, ou ácidos carboxílicos, substâncias que contém uma ou mais carboxilas em suas moléculas (PENZ Jr. et al., 1993) e que fazem referência aos ácidos fracos, de cadeia curta (C1-C7), que produzem menos prótons por molécula quando se dissociam (DIBNER \& BUTTIN, 2002). Os principais ácidos orgânicos são acético, butírico, fumárico, propiônico e fórmico, que por possuírem função antimicrobiana são utilizados há alguns anos na nutrição animal (CHERRINGTON et al., 1991). Seu uso na nutrição de leitões já é prática comum e tem como objetivo auxiliar a digestão protéica, além de controlar o desenvolvimento bacteriano intestinal (COLE et al., 1968; RISLEY et al., 1991). Em termos de desempenho, CANIBE et al. (2001) concluíram que a adição de 1,8\% de DK em dietas para leitões desmamados não alterou o consumo de ração, ganho de peso e conversão alimentar. Para PAULISCK et al. (1996), a adição de diformiato de potássio (DK) nas dietas aumentou a taxa de crescimento em suínos.

$\mathrm{Na}$ avicultura, supõe-se que a função antimicrobiana dos ácidos orgânicos tem grande importância, tendo em vista a maior capacidade de digestão protéica das aves em relação aos suínos, em idades fisiologicamente similares (NOY \& SKLAN, 1995). A adição de ácido fórmico à dieta diminuiu o $\mathrm{pH}$ do intestino delgado e também a quantidade de enterobactérias (CANIBE \& JENSEN, 2001). Tal ação se deve ao poder inibitório que possuem sobre os microorganismos, acidificando o meio e impedindo seu desenvolvimento nas matérias-primas e nas rações (PENZ Jr. et al., 1993).

O DK foi desenvolvido como alternativa ao ácido fórmico, devido aos problemas gerados durante sua manipulação (odor forte e corrosão de equipamentos durante o processamento de rações), porém existem poucos estudos sobre seus benefícios na nutrição de frangos de corte, e os resultados disponíveis não são suficientes para a determinação de seus reais efeitos sobre o desempenho das aves.

Dessa forma, o objetivo do presente trabalho foi o de avaliar os efeitos da adição de diformiato de potássio (DK) em dietas, sobre o desempenho de frangos de corte.

\section{MATERIAL E MÉTODOS}

Para a realização deste trabalho foram utilizados 1.800 frangos de corte machos da linhagem Ross 308, oriundos de matrizes com 47 semanas de idade. As aves foram alojadas aleatoriamente em dois experimentos de 900 aves cada, com cinco tratamentos com seis repetições de 30 aves, em um galpão experimental dividido em boxes, medindo 1,5m x 1,5m, recobertos com cama de maravalha, tendo iluminação artificial controlada até o $14^{\circ}$ dia de idade. No experimento 1 , as aves receberam dietas contendo doses crescentes de DK (0, 3, 6, 9 e $12 \mathrm{~kg} \mathrm{DK} \mathrm{t}^{-1}$ de ração) no período de 1 a 42 dias de idade. No experimento 2, foram utilizadas as mesmas doses de DK, porém, apenas no período de 21 a 42 dias. Até o $21^{\circ}$ dia de idade, as aves receberam a mesma dieta (a mesma utilizada no experimento, sem a adição de DK).

O manejo utilizado foi o preconizado pela indústria avícola, com água e ração à vontade, sendo as mortalidades pesadas e anotadas. Em ambos os experimentos, as aves foram pesadas ao alojamento, aos 21 e aos 42 dias de idade, para a avaliação do consumo de ração, do ganho de peso e da conversão alimentar. Os dados obtidos foram submetidos à análise de variância, e posteriormente à análise de regressão. Na tabela 1, estão apresentadas as composições calculadas das dietas experimentais utilizadas. As tabelas 2 e 3 apresentam os níveis de $\mathrm{Na}^{+}, \mathrm{K}^{+}$e $\mathrm{Cl}^{-}$, além dos balanços eletrolíticos das dietas experimentais dos experimentos 1 e 2 , respectivamente.

\section{RESULTADOS E DISCUSSÃO}

Na tabela 4, estão apresentados os resultados de consumo de ração (CR), ganho de peso (GP) e conversão alimentar (CA) das aves, para o experimento 1 . No período inicial, de 1 a 21 dias de idade, a adição de DK à dieta não teve efeito sobre o consumo de ração e a conversão alimentar das aves. Observou-se nesse período uma redução linear para ganho de peso das aves, em que o aumento da quantidade de DK às dietas determinou redução no ganho de peso. Assim como no período inicial, no período de 1 a 42 dias, a adição de DK à dieta reduziu linearmente o consumo de ração e ganho de peso, sem, no entanto, afetar a conversão alimentar das aves. Essa redução no consumo de ração e ganho de peso, se deve muito provavelmente ao aumento das doses de DK à dieta, em que quanto maior foi a dose de DKa, menor foi o consumo de ração e ganho de peso das aves. Esses resultados encontrados não estão de acordo com BORGES et al. (2003a), em que aves que 
Tabela 1 - Dietas basais.

\begin{tabular}{|c|c|c|c|}
\hline \multirow[t]{2}{*}{ Ingredientes (\%) } & \multirow[b]{2}{*}{$1-21$} & \multirow[b]{2}{*}{$22-35$} & \multirow[b]{2}{*}{$36-42$} \\
\hline & & & \\
\hline Milho & 57,10 & 59,77 & 63,58 \\
\hline Farelo de soja & 34,48 & 31,38 & 28,13 \\
\hline Óleo de soja & 3,13 & 3,77 & 3,44 \\
\hline Fosfato Bicálcico & 1,78 & 1,64 & 1,44 \\
\hline Calcário & 0,96 & 0,92 & 0,93 \\
\hline Sal comum & 0,42 & 0,39 & 0,36 \\
\hline Premix vitamínico & 0,30 & 0,30 & 0,30 \\
\hline Premix mineral & 0,20 & 0,20 & 0,20 \\
\hline DL-metionina & 0,22 & 0,19 & 0,17 \\
\hline L-lisina (HCl) & 0,16 & 0,16 & 0,17 \\
\hline L-treonina & 0,01 & 0,03 & 0,03 \\
\hline Inerte & 1,20 & 1,20 & 1,20 \\
\hline Proteína bruta (\%) & 20,87 & $\begin{array}{c}\text { ição cal } \\
19,69\end{array}$ & 18,52 \\
\hline Energia metabolizável (kcal kg-1) & 3.000 & 3.075 & 3.100 \\
\hline $\mathrm{Ca}(\%)$ & 0,93 & 0,88 & 0,82 \\
\hline P disponível (\%) & 0,44 & 0,41 & 0,37 \\
\hline $\mathrm{Na}(\%)$ & 0,18 & 0,17 & 0,16 \\
\hline $\mathrm{Cl}(\%)$ & 0,32 & 0,31 & 0,29 \\
\hline Lisina Dig. (\%) & 1,11 & 1,04 & 0,97 \\
\hline Metionina Dig. (\%) & 0,51 & 0,47 & 0,43 \\
\hline Metionina+Cistina Dig. (\%) & 0,79 & 0,74 & 0,69 \\
\hline Treonina Dig. (\%) & 0,71 & 0,69 & 0,65 \\
\hline Triptofano Dig. (\%) & 0,23 & 0,21 & 0,19 \\
\hline
\end{tabular}

Os níveis de inclusão de DK foram realizados em substituição parcial ou total à areia lavada.

Tabela 2 - Níveis de $\mathrm{Na}^{+}, \mathrm{K}^{+}$, $\mathrm{Cl}^{-}$e balanço eletrolítico (BE) das dietas experimentais utilizadas no experimento 1.

\begin{tabular}{|c|c|c|c|c|}
\hline & $\mathrm{Na}^{+}(\%)$ & $\mathrm{K}^{+}(\%)$ & $\mathrm{Cl}^{-}(\%)$ & $\mathrm{BE}\left(\mathrm{mEq} \mathrm{kg}{ }^{-1}\right)$ \\
\hline $\mathrm{T} 1$ (0kg de DK t $\left.{ }^{-2}\right)$ & 0,216 & 0,791 & 0,046 & 283 \\
\hline $\mathrm{T} 2$ (3kg de $\mathrm{DK} \mathrm{t}^{-2}$ ) & 0,216 & 0,881 & 0,046 & 306 \\
\hline T3 (6kg de DK t ${ }^{-2}$ ) & 0,216 & 0,971 & 0,046 & 329 \\
\hline T4 (9kg de DK t ${ }^{-2}$ ) & 0,216 & 1,061 & 0,046 & 352 \\
\hline T5 (12kg de DK t $\left.{ }^{-2}\right)$ & 0,216 & 1,151 & 0,046 & 375 \\
\hline T1 (0kg de DK t $\left.{ }^{-2}\right)$ & 0,203 & 0,742 & 0,046 & 265 \\
\hline $\mathrm{T} 2$ (3kg de $\mathrm{DK} \mathrm{t}^{-2}$ & 0,203 & 0,832 & 0,046 & 288 \\
\hline T3 (6kg de DK t $\left.{ }^{-2}\right)$ & 0,203 & 0,922 & 0,046 & 311 \\
\hline T4 (9kg de DK t $\left.{ }^{-2}\right)$ & 0,203 & 1,012 & 0,046 & 334 \\
\hline T5 (12kg de DK t $\left.{ }^{-2}\right)$ & 0,203 & 1,102 & 0,046 & 357 \\
\hline $\mathrm{T} 1$ (0kg de DK t ${ }^{-2}$ ) & 0,192 & $\begin{array}{l}42 \text { dias- } \\
0,693\end{array}$ & 0,079 & 238 \\
\hline $\mathrm{T} 2$ (3kg de $\mathrm{DK} \mathrm{t}{ }^{-2}$ ) & 0,192 & 0,783 & 0,079 & 261 \\
\hline T3 (6kg de DK t $\left.{ }^{-2}\right)$ & 0,192 & 0,873 & 0,079 & 284 \\
\hline T4 (9g de DK t ${ }^{-2}$ ) & 0,192 & 0,963 & 0,079 & 307 \\
\hline T5 (12kg de DK t $\left.{ }^{-2}\right)$ & 0,192 & 1,053 & 0,079 & 330 \\
\hline
\end{tabular}

Ciência Rural, v.39, n.8, nov, 2009. 
Tabela 3 - Níveis de $\mathrm{Na}^{+}, \mathrm{K}^{+}, \mathrm{Cl}^{-}$e balanço eletrolítico (BE) das dietas experimentais utilizadas no experimento 2 .

\begin{tabular}{|c|c|c|c|c|}
\hline $\mathrm{T} 1$ (0kg de $\left.\mathrm{DK} \mathrm{t}^{-2}\right)$ & 0,203 & 0,744 & 0,046 & 265 \\
\hline $\mathrm{T} 2$ (3kg de DK t ${ }^{-2}$ ) & 0,203 & 0,834 & 0,046 & 288 \\
\hline T3 (6kg de DK t ${ }^{-2}$ ) & 0,203 & 0,924 & 0,046 & 311 \\
\hline $\mathrm{T} 4$ (9kg de $\left.\mathrm{DK} \mathrm{t}{ }^{-2}\right)$ & 0,203 & 1,014 & 0,046 & 334 \\
\hline T5 (12g de DK t $\left.{ }^{-2}\right)$ & 0,203 & 1,104 & 0,046 & 357 \\
\hline \multicolumn{5}{|c|}{-------------------------De 36 a 42 dias----- } \\
\hline $\mathrm{T} 1$ (0kg de DK t $\left.{ }^{-2}\right)$ & 0,192 & 0,693 & 0,079 & 238 \\
\hline $\mathrm{T} 2$ (3kg de $\left.\mathrm{DK} \mathrm{t} \mathrm{t}^{-2}\right)$ & 0,192 & 0,783 & 0,079 & 261 \\
\hline T3 (6kg de DK t ${ }^{-2}$ ) & 0,192 & 0,873 & 0,079 & 284 \\
\hline $\mathrm{T} 4$ (9kg de $\mathrm{DK} \mathrm{t}^{-2}$ ) & 0,192 & 0,963 & 0,079 & 307 \\
\hline T5 (12g de DK t $\left.{ }^{-2}\right)$ & 0,192 & 1,053 & 0,079 & 330 \\
\hline
\end{tabular}

receberam dietas contendo DK melhoraram o desempenho zootécnico. Em outro trabalho, BORGES et al. (2003b) também verificaram que a utilização de sais via água de bebida ou ração, melhorou o desempenho de frangos de corte, reduzindo as perdas ocasionadas pelo estresse calórico. Da mesma forma, TEETER \& SMITH (1986), submetendo frangos de corte a condições de estresse calórico $\left(35^{\circ} \mathrm{C}\right.$ e $70 \%$ UR), observaram um melhor desempenho zootécnico de frangos de corte quando utilizaram dietas contendo $0,88 \%$ de $\mathrm{K}$.
Os resultados encontrados neste experimento podem ser justificados em razão das adições de doses crescentes de diformiato de potássio $\left(0,3,6,9\right.$ e $\left.12 \mathrm{~kg} \mathrm{DK} \mathrm{t}^{-1}\right)$ aumentarem as concentrações de potássio nas dietas, variando de $0,69 \%$ a $1,14 \%$, e tendo como consequência os efeitos negativos sobre o consumo de ração e o ganho de peso das aves. Quando há excessos de potássio, o organismo deixa de eliminar prótons para eliminar potássio, o que levaria o organismo à alcalose, ocorrendo como resposta fisiológica, a inibição do consumo e conseqüentemente o menor ganho de peso (GRANNER, 1998).

Na tabela 5, são apresentados os resultados do experimento 2, no qual as aves foram alimentadas com dietas contendo DK apenas no período de 21 a 42 dias. Os resultados encontrados foram semelhantes aos do experimento 1. Analisando os dados, observase que ocorreu uma redução do consumo de ração e do ganho de peso, não sendo observado nenhum efeito da adição de DK sobre a conversão alimentar das aves. Resultados semelhantes foram encontrados por SELLE et al. (2004), os quais observaram que a adição de 4,5g de $D K t^{-1}$ de ração não causou alteração no desempenho de frangos de corte aos 33 dias de idade. O efeito esperado do uso do DK se baseia na melhoria da conversão alimentar e do ganho de peso, além da melhoria da integridade intestinal das aves (BASF,

Tabela 4 - Consumo de ração (CR), ganho de peso (GP) e conversão alimentar (CA) de frangos de corte alimentados com dietas contendo níveis crescentes de diformiato de potássio, no período de 1 a 42 dias de idade (Experimento 1).

\begin{tabular}{|c|c|c|c|}
\hline Diformiato $\left(\mathrm{kg} \mathrm{t}^{-1}\right)$ & CR (g) & GP (g) & $\mathrm{CA}(\mathrm{g} / \mathrm{g})$ \\
\hline 0 & 1.070 & 811 & 1,315 \\
\hline 3 & 1.070 & 800 & 1,337 \\
\hline 6 & 1.063 & 788 & 1,349 \\
\hline 9 & 1.047 & 762 & 1,373 \\
\hline 12 & 1.045 & 763 & 1,373 \\
\hline $\mathrm{P}$ & 0,931 & 0,001 & 0,633 \\
\hline Equação de regressão & NS & $Y=812-4,49 X$ & NS \\
\hline$r^{2}$ & - & 0,93 & - \\
\hline CV (\%) & 6,11 & 3,11 & 5,58 \\
\hline Diformiato $\left(\mathrm{kg} \mathrm{t}^{-1}\right)$ & CR (g) & GP (g) & $\mathrm{CA}(\mathrm{g} / \mathrm{g})$ \\
\hline 0 & 4.225 & 2.528 & 1,671 \\
\hline 3 & 4.116 & 2.437 & 1,689 \\
\hline 6 & 4.121 & 2.440 & 1,689 \\
\hline 9 & 4.101 & 2.427 & 1,69 \\
\hline 12 & 4.035 & 2.403 & 1,679 \\
\hline $\mathrm{P}$ & 0,033 & 0,009 & 0,867 \\
\hline Equação de regressão & $Y=4199-13,11 X$ & $Y=2499-8,66 X$ & NS \\
\hline$r^{2}$ & 0,83 & 0,74 & - \\
\hline CV (\%) & 3,33 & 2,3 & 2,18 \\
\hline
\end{tabular}

Ciência Rural, v.39, n.8, nov, 2009. 
Tabela 5 - Consumo de ração (CR), ganho de peso (GP) e conversão alimentar (CA) de frangos de corte alimentados com dietas contendo níveis crescentes de diformiato de potássio, no período de 21 a 42 dias (Experimento 2).

\begin{tabular}{lccc}
\hline Diformiato $\left(\mathrm{kg} \mathrm{t}^{-1}\right)$ & CR $(\mathrm{g})$ & GP $(\mathrm{g})$ & CA $(\mathrm{g})$ \\
\hline 0 & 3.157 & 1.724 & 1,831 \\
3 & 3.075 & 1.689 & 1,823 \\
6 & 3.094 & 1.686 & 1,835 \\
9 & 3.080 & 1.667 & 1,847 \\
12 & 2.953 & 1.602 & 1,844 \\
$\mathrm{P}$ & 0,0092 & 0,052 & 0,876 \\
Equação de regressão & $\mathrm{Y}=3152-13,40 \mathrm{X}$ & $\mathrm{Y}=1727-8,84 \mathrm{X}$ & $\mathrm{NS}$ \\
$\mathrm{r}^{2}$ & 0,74 & 0,87 & 0,64 \\
$\mathrm{CV}(\%)$ & 3,29 & 3,99 & 2,35 \\
\hline
\end{tabular}

2005). Nesse contexto, FRANCO et al. (2005), trabalhando com integridade intestinal de frangos de corte, observaram efeitos benéficos com uso de diformiato de potássio sobre a mucosa intestinal de frangos de corte. Em um estudo com suínos, OVERLAND \& GRANLI (2001) avaliaram o uso de DK como promotor de crescimento, e concluíram que o modo de ação do diformiato de potássio se baseia na redução da população microbiana maléfica, como $\boldsymbol{E}$. coli e Salmonella.

Os efeitos negativos observados sobre 0 desempenho das aves neste experimento podem ter sido gerados pelos elevados índices de balanço eletrolítico, causados pelas altas concentrações de potássio nas dietas, visto que as dietas controle, à base de milho e farelo de soja, já continham balanço eletrolítico muito próximo daquele considerado ideal. No presente estudo o balanço eletrolítico para os grupos tratado com DK, variou de 288 até 374, ultrapassando assim os padrões ótimos recomendados, próximos de 250meq $\mathrm{kg}^{-1}$ de ração (MONGIN \& SAUVEUR, 1977; AIT-BOULAHSEN et al., 1989; BORGES et al., 2003b; BORGES et al., 2007).

Trabalhando com balanço eletrolítico, BORGES et al. (2003a) constataram que a relação eletrolítica da ração $(\mathrm{Na}+\mathrm{K}+\mathrm{Cl}-)$ interfere no desempenho das aves, e a relação ideal varia segundo o eletrólito manipulado, estando entre 207 e $236 \mathrm{mEq}$ $\mathrm{kg}^{-1}$ de ração. Em outro estudo, BORGES et al. (1999), concluíram que relações eletrolíticas elevadas (354 e $360 \mathrm{mEq} \mathrm{kg}{ }^{-1}$ ) obtidas pela suplementação de K ou de $\mathrm{Na}$ na ração deprimiram o crescimento das aves. Segundo os autores, os níveis de K e Na nessas rações teriam ultrapassado a tolerância da ave, já que as exigências para esses minerais são estimadas em 0,30 e 0,20\%, respectivamente (NRC, 1994).

\section{CONCLUSÃO}

Nas condições experimentais, pode-se concluir que a adição de diformiato de potássio às dietas de frangos de corte reduz o consumo de ração e o ganho de peso das aves, sem causar efeitos sobre a conversão alimentar das aves.

\section{REFERÊNCIAS}

AIT-BOULAHSEN, A. et al. Effect of fasting and acute heat stress on body temperature, blood acid-base and electrolyte status in chickens. Comparative Biochemistry Physiology, v.94, n.4, p.683-687, 1989. Disponível em: <http://dx.doi.org/ 10.1016/0300-9629(89)90617-8>. Acesso em: 21 jan. 2009. doi: 10.1016/0300-9629(89)90617-8.

BORGES, S.A. et al. Balanço eletrolítico em dieta pré-inicial de frangos de corte durante o verão. Revista Brasileira de Ciência Avícola, v.1, p.175-179, 1999.

BORGES, S.A. et al. Dietary electrolyte balance for broiler chickens under moderately high ambient temperatures and humidities relatives. Poultry Science, v.82, p.301-308, 2003a.

BORGES, S.A. et al. Fisiologia do estresse calórico e a utilização de eletrólitos em frangos de corte. Ciência Rural, v. 33, n.5, p. 975-981, 2003b. Disponível em: <http://www.scielo.br/ scielo.ph p ? s cript = sci_art text \& pid = S 0103 $84782003000500028 \& \operatorname{lng}=p t \& n r m=i s o \& t \operatorname{lng}=p t>$. Acesso em: 21 jan. 2009. doi: 10.1590/S0103-84782003000500028.

BORGES, S.A. et al. Acid-base balance in broilers. World's Poultry Science Journal, v.63, p.73-81, 2007.

BASF Nutrição Animal. Formi ${ }^{\circledR}$ : sinal verde para uma avicultura sem fronteiras. São Paulo, 2005. (Folheto técnico).

CANIBE, N.; JENSEN, B.B. Effect of feed structure and processing and addition of addition of formic acid to the feed on the microbial ecology of the gastrointestinal tract of pigs. In.: WORKSHOP ON ALTERNATIVES TO FEED ANTIBIOTICS AND ANTICOCCIDIAL IN THE PIG AND POULTRY MEAT PRODUCTION, 2001, Oslo. Proceedings... Oslo: AFAC, 2001. p.1-5. 
CANIBE, N. et al. Effect of K-diformate in starter diets on acidity, microbiota, and the amount of organic acids in the digestive tract of piglets, and on gastric alterations. Journal of Animal Science, v.79, p.2123-2133, 2001.

CHERRINGTON, C.A. et al. Organic acids: chemistry, antibacterial activity and practical applications. Advances Microbiological Physiology, v.32, p.87-108. 1991. Disponível em: <http://dx.doi.org/10.1016/S00652911(08)60006-5>. Acesso em: 21 jan. 2009. doi: 10.1016/ S0065-2911(08)60006-5.

COLE, D.J.A. et al. The effect on performance and bacterial flora of lactic acid, propionic acid, calcium propionate and calcium acrylate in the drinking water of weaned pigs. Veterinary Record, v.83, p.459-464, 1968.

DIBNER, J.J.; BUTTIN, P. Use of organic acids as a model to study the impact of gut microflora on nutrition and metabolism. Journal Applied of Poultry Research, v.11, p.453-463, 2002.

FRANCO, J.R.G. et al. Efeito da utilização do ácido orgânico diformiato de potássio sobre a morfometria do intestino de frangos de corte. In: CONFERÊNCIA APINCO DE CIÊNCIA E TECNOLOGIA AVÍCOLAS, 2005, Santos. Anais... Campinas: FACTA, 2005. V.7, p.47-47.

GRANNER, K.D. Hormônios da córtex da adernal. In: HARPER, J.C. (Ed.). Bioquímica. 8.ed. São Paulo: Atheneu, 1998. p.707-709.

MONGIN, P.; SAUVEUR, B. Interrelationships between mineral nutrition, acid-base balance, growth and cartilage abnormalities. Poultry Science, v.12, p.235-247, 1977.

NOY, Y.; SKLAN, D. Digestion and absorption in the young chick. Poultry Science, v.74, p.366-373, 1995.
National Research Council. Nutrient requirements of poultry. 9.ed. Washington: National Academy, 1994. 176p.

OVERLAND, M.; GRANLI, T. Potassium diformate (Formi ${ }^{\circledR}$ ) - Effect on gut microflora and growth performance. In: WORKSHOP ON ALTERNATIVES TO FEEDS ANTIBIOTICS AND ANTICOCCIDIAL IN THE PIG AND POULTRY MEAT PRODUCTION, 2001, Oslo. Proceedings... Oslo: AFAC, 2001. p.6-10.

AULISCK, B.R. et al. Dosis effects of potassium diformate (Formi ${ }^{\mathrm{TM}} \mathrm{LHS}$ ) on the performance of growing piglets. Agrobiology Research, v.12, p.1-30, 1996.

PENZ Jr., A.M. et al. Ácidos orgânicos na alimentação de aves. In: CONFERÊNCIA APINCO DE CIÊNCIA E TECNOLOGIA AVÍCOLAS, 1993, Santos. Anais... Campinas: FACTA, 1993. p.111-119.

RISLEY, C.R. et al. Effects of organic acids with and without a microbial culture on performance and gastrointestinal tract measurements of weanling pigs. Animal Feed Science and Technology, v.35, p.259-270, 1991. Disponível em: <http:// dx.doi.org/10.1016/0377-8401(91)90132-C >. Acesso em: 21 jan. 2009. doi: 10.1016/0377-8401(91)90132-C.

SELLE, P.H. et al. Effects of postassium diformate inclusion in broiler diets on growth performance and nutrient utilisation. Australian Poultry Science Symposium, v.16, p.55-58, 2004.

TEETER, R.G.; SMITH, M.O. High chronic ambient temperature stress effects on broiler acid-basic and their response to supplemental ammonium chloride, potassium chloride and potassium carbonate. Poultry Science, v.65, p.1777-1781, 1986 